\title{
Special issue on computational intelligence-based modeling, control and estimation in modern mechatronic systems
}

\author{
Hai Wang ${ }^{1} \cdot$ Jinchuan Zheng $^{2} \cdot$ Yuqian $\mathrm{Lu}^{3} \cdot$ Shihong Ding $^{4} \cdot$ Hicham Chaoui $^{5}$ \\ Received: 2 December 2021 / Accepted: 2 December 2021 / Published online: 30 January 2022 \\ (C) The Author(s), under exclusive licence to Springer-Verlag London Ltd., part of Springer Nature 2021
}

Modern mechatronic systems are currently experiencing immense changes in the fourth industrial revolution with the recent advances in artificial intelligence (AI) techniques, big data analytics, cutting-edge telecommunication technologies, control theory and microelectronics. Mechatronic systems become highly multidisciplinary with an ever-increasing synergistic integration of mechanical, electrical/electronic, control and information disciplines. The complex technical changes urge the modern mechatronic systems to exhibit more stable and excellent operating performance, in terms of strong robustness and reliability, design simplicity and smartness. However, mechatronic systems are continuously facing technical challenges and difficulties, under the parametric and/or structural uncertainties, undesired external disturbances, fast-varying references, sensor noises, nonlinearities, mechanical and mechatronic component failures and/or restricted online computing time of the control execution. In order to further address the above concerns and improve the overall performance of mechatronic systems, many computational intelligence (CI) technologies (fuzzy logic, neural networks, reinforcement learning, AI, etc.) have been popularly utilized to assist with the modeling, control and estimation of mechatronic systems, which attract

Hai Wang

hai.wang@murdoch.edu.au

1 Discipline of Engineering and Energy, Centre for WaterEnergy-Waste of Harry Butler Institute, Murdoch University, Perth WA6150, Australia

2 Faculty of Science, Engineering and Technology, Swinburne University of Technology, Melbourne, VIC 3122, Australia

3 Department of Mechanical Engineering, The University of Auckland, Auckland 1010, New Zealand

4 School of Electrical and Information Engineering, Jiangsu University, Zhenjiang 212013, China

5 Department of Electronics, Carleton University, Ottawa, Canada considerable attention among researchers and engineers from academia and industry. Meanwhile, the latest developments of sensors, microcontrollers, DSP, FPGA, etc., also enhance the real-time CI-based control and estimation implementations of mechatronic systems.

The submitted manuscripts were reviewed by experts from both academia and industry. After two to three rounds of reviewing, the highest quality papers were accepted for this special issue. This special issue will be published by Neural Computing and Applications as special issues. Totally, 20 papers are suggested to $\mathrm{EiC}$ for acceptance. The selected papers are summarized as follows.

Jung et al. [1] propose weighted recursive Gaussian process (WRPG) to overcome the effect of arm position change in estimating a subject's wrist movement of biomechatronic systems. An adaptive backstepping integral nonsingular terminal sliding mode control (BINTSMC) based on extreme learning machine (ELM) for trajectory tracking control of robotic manipulators is proposed by Gao et al. [2]. MahmoudZadeh et al. [3] present an efficient data collection strategy exploiting a team of unmanned aerial vehicles (UAVs) to monitor and collect the data of a large distributed sensor network usually used for environmental monitoring, meteorology, agriculture and renewable energy applications. Tu et al. [4] study a novel scheme for the tracking problem of nonlinear systems, where two reinforcement learning algorithms are proposed to design the optimal control law. Tang el al. [5] introduce a deep neural network (DNN)-based hierarchical learning optimization method to establish an online approach to focused coordination dispatch problems in multi-regional power grid with interconnected tie-lines. Lan et al. [6] propose an integrated condition monitoring method combining model-based fault diagnosis and data-driven prognosis is proposed for steer-by-wire (SBW) system using optimized ELM (OELM). Yang et al. [7] focus on the finite-time lag synchronization (FTLS) of uncertain complex networks involving impulsive disturbance effects. A radial basis function (RBF) neural network-based integral 
terminal sliding mode controller for tackling the problem of attitude stabilization control of spacecraft under angular velocity constraint is investigated in Yu et al. [8]. Jin et al. [9] adopt an adaptive neural network (NN)-based control technique to deal with the nonlinearities and uncertainties for the trajectory tracking to achieve finite-time control of wheeled robotic systems with nonlinear dynamics and uncertainties. Sun et al. [10] present a double-hidden-layer output feedback neural network fast nonsingular terminal sliding mode control strategy for path-tracking tasks of autonomous vehicles.

Wang et al. [11] use a novel concurrent semi-supervised model to estimate the remaining useful life of the aeroengine. Ping et al. [12] use the neural network (NN) to approximate the solution of the discrete regulator equations (DREs) for tackling the tracking problem of the inverted pendulum on a cart (IPC) system. Li et al. [13] use iterative learning scheme to track the fault signals in a class of nonlinear nonrepetitive systems that subject to iterationdependent references. Fathi et al. [14] evaluate the performance and suitability of four different metaheuristic algorithms for optimal sizing of standalone microgrids in remote area. Tan et al. [15] propose a novel algorithm based on minimizing the area of a boundary enclosing partial scan data points for detecting both the pose and assembly of tubular joints with the aid of reference ideal models. Yan et al. [16] study the evasion guidance for airbreathing hypersonic vehicles (AHVs) against unknown pursuer dynamics, where the gradient descent is employed for parameter estimation of the unknown dynamics of the pursuer. Fang et al. [17] present a method for minimizing the power consumption of a data-center cooling system by optimizing the airflow pattern and the supplied cold air temperature simultaneously. Hu et al. [18] use ELM to learn changing rate of lumped uncertainties in an adaptive full order terminal sliding mode-based electronic throttle valve control system. Mohammadi et al. [19] present a model-free integral reinforcement learning (RL) strategy for nonlinear autonomous underwater vehicles (AUVs) that subject to multi-asymmetric constrained inputs. Zheng et al. [20] study an ELM based field-oriented feedback linearization speed control (ELMFOFLC) to enhance the robustness and tracking performance of a permanent magnetic synchronous motor (PMSM) system.

Acknowledgements The guest editors would like to thank Prof. John MacIntyre who is the Editor in Chief of Neural Computing and Applications. The guest editors would like to thank the reviewers for their high-quality reviews, which provide insightful and constructive feedback to the authors of the papers. The guest editors also would like to thank Journal Editors, Rachel Moriarty, Deepika Sureshkumar, and Rashmi Jenna, for their help on submission and publication.

\section{References}

1. Jung MC, Chai R, Zheng J, Nguyen H (2021) Enhanced myoelectric control against arm position change with weighted recursive Gaussian process. Neural Comput Appl. https://doi.org/ 10.1007/s00521-021-05743-y

2. Gao M-M, Jin X-Z, Ding L-J (2021) Robust adaptive backstepping INTSM control for robotic manipulators based on ELM. Neural Comput Appl. https://doi.org/10.1007/s00521-021-05824y

3. MahmoudZadeh S, Yazdani A, Elmi A et al (2021) Exploiting a fleet of UAVs for monitoring and data acquisition of a distributed sensor network. Neural Comput Appl. https://doi.org/10.1007/ s00521-021-05906-X

4. Tu Y, Fang H, Yin Y, He S (2021) Reinforcement learning-based nonlinear tracking control system design via LDI approach with application to trolley system. Neural Comput Appl. https://doi. org/10.1007/s00521-021-05909-8

5. Tang H, Lv K, Bak-Jensen B et al (2021) Deep neural networkbased hierarchical learning method for dispatch control of multiregional power grid. Neural Comput Appl. https://doi.org/10. 1007/s00521-021-06008-4

6. Lan D, Yu M, Huang Y et al (2021) Fault diagnosis and prognosis of steer-by-wire system based on finite state machine and extreme learning machine. Neural Comput Appl. https://doi.org/10.1007/ s00521-021-06028-0

7. Yang X, Li X, Duan P (2021) Finite-time lag synchronization for uncertain complex networks involving impulsive disturbances. Neural Comput Appl. https://doi.org/10.1007/s00521-021-059878

8. Yu B, Du H, Ding L et al (2021) Neural network-based robust finite-time attitude stabilization for rigid spacecraft under angular velocity constraint. Neural Comput Appl. https://doi.org/10.1007/ s00521-021-06056-w

9. Jin X, Zhao Z, Wu X et al (2021) Adaptive NN-based finite-time trajectory tracking control of wheeled robotic systems. Neural Comput Appl. https://doi.org/10.1007/s00521-021-06021-7

10. Sun Z, Zou J, He D, Zhu W (2021) Path-tracking control for autonomous vehicles using double-hidden-layer output feedback neural network fast nonsingular terminal sliding mode. Neural Comput Appl. https://doi.org/10.1007/s00521-021-06101-8

11. Wang T, Guo D, Sun X-M (2021) Remaining useful life predictions for turbofan engine degradation based on concurrent semi-supervised model. Neural Comput Appl. https://doi.org/10. 1007/s00521-021-06089-1

12. Ping Z, Zhou M, Liu C et al (2021) An improved neural network tracking control strategy for linear motor-driven inverted pendulum on a cart and experimental study. Neural Comput Appl. https://doi.org/10.1007/s00521-021-05986-9

13. Li F, Kenan D, Shuiqing $X$ et al (2021) A nonrepetitive fault estimation design via iterative learning scheme for nonlinear systems with iteration-dependent references. Neural Comput Appl. https://doi.org/10.1007/s00521-021-06176-3

14. Fathi M, Khezri R, Yazdani A, Mahmoudi A (2021) Comparative study of metaheuristic algorithms for optimal sizing of standalone microgrids in a remote area community. Neural Comput Appl. https://doi.org/10.1007/s00521-021-06165-6

15. Tan YZ, Pang CK, Al Mamun A et al (2021) Precise pose and assembly detection of generic tubular joints based on partial scan data. Neural Comput Appl. https://doi.org/10.1007/s00521-02106246-6

16. Yan T, Cai Y, Xu B (2021) Evasion guidance for air-breathing hypersonic vehicles against unknown pursuer dynamics. Neural Comput Appl. https://doi.org/10.1007/s00521-021-06250-w 
17. Fang Q, Zhou J, Wang S, Wang Y (2021) Control-oriented modeling and optimization for the temperature and airflow management in an air-cooled data-center. Neural Comput Appl. https://doi.org/10.1007/s00521-021-06385-w

18. Hu Y, Wang H, Yazdani A, Man Z (2021) Adaptive full order sliding mode control for electronic throttle valve system with fixed time convergence using extreme learning machine. Neural Comput Appl. https://doi.org/10.1007/s00521-021-06365-0

19. Mohammadi M, Arefi MM, Vafamand N, Kaynak O (2021) Control of an AUV with completely unknown dynamics and multi-asymmetric input constraints via off-policy reinforcement learning. Neural Comput Appl. https://doi.org/10.1007/s00521021-06476-8

20. Zheng Y, Cao Z, Wang S et al (2021) Extreme learning machine based field-oriented feedback linearization speed control of permanent magnetic synchronous motors. Neural Comput Appl. https://doi.org/10.1007/s00521-021-06722-z

Publisher's Note Springer Nature remains neutral with regard to jurisdictional claims in published maps and institutional affiliations. 